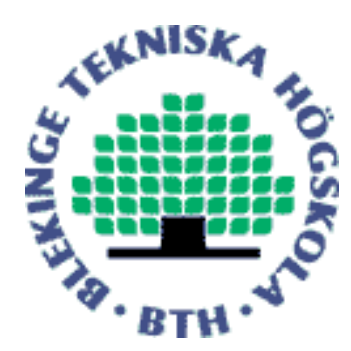

Electronic Research Archive of Blekinge Institute of Technology http://www.bth.se/fou/

This is an author produced version of a paper published in Nonlinear Analysis: Hybrid Systems. This paper has been peerreviewed but may not include the final publisher proof-corrections or journal pagination.

Citation for the published paper:

Jan Melin, Anders Hultgren, Torsten Lindström.

"Two types of limit cycles of a resonant converter modelled by a three-dimensional system".

Nonlinear Analysis: Hybrid Systems 2008, Vol. 2:4, pp. 1275-1286

DOI: 10.1016/j.nahs.2008.09.020

Access to the published version may require subscription.

Published with permission from:

Elsevier 


\title{
Two types of Limit Cycles of a Resonant Converter modelled by a three-dimensional system
}

\author{
Jan Melin \\ School of Pure and Applied Natural Sciences \\ University of Kalmar \\ SE-39182 KALMAR \\ Anders Hultgren \\ Department of Signal Processing \\ School of Engineering \\ Blekinge Institute of Technology \\ SE-37225 Ronneby \\ Torsten Lindström \\ School of Pure and Applied Natural Sciences \\ University of Kalmar \\ SE-39182 KALMAR
}

September 16, 2008

\begin{abstract}
In this paper the long-run dynamics of a resonant converter is studied. The modelling of the resonant converter can be done using various approximations and the long-run dynamics of the two dimensional cases were earlier analyzed. In this paper we discuss results that can be extended to the three dimensional case. It seems that
\end{abstract}


limit cycles describe the long-run dynamics in important situations in the three dimensional case, too. Our objective is to relate the limit cycles found previously in the two-dimensional case to those found now in the three dimensional approximation.

Keywords: Piecewise linear system, plane, subspace, limit cycle, projection. AMS Subject Classification(2000): 34A36, 34A12, 34H05.

\section{Introduction}

Modelling of physical systems with abrupt changes in the dynamics as switched systems has gained a large interest during the last decade. Analysis of such systems is difficult due to the non-smooth changes of dynamics. A switched system can show phenomena like sliding mode and chattering. In the analysis work of applying switched models in industrial control systems it is important to characterize the global behaviour of the systems not to excite chattering behaviour or non favourable limit cycles.

In this article a controlled resonant electrical power converter is analyzed. See Figure 1. Switched electrical power converters are widespread and used for almost every electrically driven system. Modelling a switched converter as a switched system enables to get faster and more accurate control compared to averaged models and control. In this analysis a resonant power converter is modelled as a switched system and it is controlled by a switched controller. In Hultgren and Lenells [11] and Melin and Hultgren [18] a detailed description of the electrical converter is given. In the latter reference also an analysis of a second order switched model of the system is given. The controlled second order system was found to have nine different solution sets. Limit cycles analysis for systems controlled by switch controllers using different methods, can be found in Angulo et al [5], Flieller et al [3], diBernardo-Camlibel [4] and X.-S. Yang-G. Chen [20].

In this paper the converter is modelled as a third order switched system and controlled by switch surfaces making the set of possible solutions even richer. Our switched model is a piecewise linear system. A general framework for how such systems should be studied has been given by Filippov [6]. In our case the switching surfaces consist of one cylinder and three planes, two of the planes are parallel to each other and perpendicular to the main axis of the cylinder. The last plane contains the main axis of the cylinder and is therefore perpendicular to the other planes, see Figure 2. 
Two-dimensional approximations describing resonant converters by switched systems have been studied previously by Melin and Hultgren [18]. Generally, extensions of the classical Poincaré-Bendixson theory (e. g. Hirsch and Smale [8]) and extensions of currently existing tools for estimating the number of limit cycles from above (e. g. Ye et. al. [21] and Zhang et. al. [22]) have been used in order to analyze number of limit cycles that exist in such switched systems. The extensions referred to above are based on distribution theory following Hörmander [9], cf. Melin [15, 14, 16]. Attempts to extend various parts of Poincaré-Bendixson theory into higher dimensions exist, cf. the extensions of the Bendixson criterion by Li and Muldowney $[13,12]$ which also include non-smooth cases (Hou [10]). In some cases one may use theory of monotone dynamical systems in order to reduce dimension of the system under study, see e. g. Smith [19].

It turns out that our system allows for dimension reductions, too. More precisely, we show that trajectories essential for limit cycle behavior in the three-dimensional approximation of the resonant converter may be projected onto trajectories that are close to the two-dimensional approximation of the same system.

Our paper is organized as follows. Section 2 describes the model and its basic properties. Certain restriction on its parameter values and standard values used in our numerical simulations are mentioned here. The section ends up with a remark about essential symmetries present in the system. We continue in section 3 by describing the structure or possible non-wandering sets of the model. It turns out that the non-wandering sets may consist of fixed points, limit cycles, and som more complicated unions of trajectories. We go on analyzing the limit cycles encountered in two main cases. The first case consists of limit cycles entirely inside the switching surfaces defined by the cylinder, introduced in section 4, and the second case consist of a special type of limit cycles that occur both inside and outside the cylinder, introduced in section 5. Both of these cases are studied through projections onto two-dimensional systems close to the two-dimensional approximation analyzed previously by Melin and Hultgren [18]. We summarize the results in section 6 and indicate some ideas for future research. 


\section{The model}

Our three dimensional approximation of the resonant converter takes the form

$$
\left(\begin{array}{c}
\dot{x} \\
\dot{y} \\
\dot{z}
\end{array}\right)=\left(\begin{array}{c}
y / C \\
-\left(x+R \cdot y+z-u_{i}\right) / L \\
\Gamma_{i} \cdot y
\end{array}\right)
$$

in case of constant load voltage $U_{0}$ with $u_{C}=x, i_{L}=y$ and $u_{w}=z$ where $i=1,2, \ldots, 12$. The corresponding switching regions are given by:

$$
\begin{aligned}
& \Omega_{1}: x^{2}+y^{2}<i_{r}^{2}, y>0,|z|<U_{0}, \\
& \Omega_{2}: x^{2}+y^{2}<i_{r}^{2}, y<0,|z|<U_{0}, \\
& \Omega_{3}: x^{2}+y^{2}>i_{r}^{2}, y>0,|z|<U_{0}, \\
& \Omega_{4}: x^{2}+y^{2}>i_{r}^{2}, y<0,|z|<U_{0}, \\
& \Omega_{5}: x^{2}+y^{2}<i_{r}^{2}, y>0, z>U_{0}, \\
& \Omega_{6}: x^{2}+y^{2}<i_{r}^{2}, y<0, z>U_{0}, \\
& \Omega_{7}: x^{2}+y^{2}>i_{r}^{2}, y>0, z>U_{0}, \\
& \Omega_{8}: x^{2}+y^{2}>i_{r}^{2}, y<0, z>U_{0}, \\
& \Omega_{9}: x^{2}+y^{2}<i_{r}^{2}, y>0, z<-U_{0}, \\
& \Omega_{10}: x^{2}+y^{2}<i_{r}^{2}, y<0, z<-U_{0}, \\
& \Omega_{11}: x^{2}+y^{2}>i_{r}^{2}, y>0, z<-U_{0}, \\
& \Omega_{12}: x^{2}+y^{2}>i_{r}^{2}, y<0, z<-U_{0} .
\end{aligned}
$$

The reference current $i_{r}>0$ is the setpoint of the controller. It represents, although as a nonlinear function, the rectified output current of the system. The switching curves for the controller can be chosen in different ways. For a discussion of different switched controllers for a resonant converter see Hultgren et al (2002) [1].

The control parameters take the values

$$
u_{i}= \begin{cases}E-U_{0}, & i=1,5,9 \\ U_{0}-E, & i=2,6,10 \\ -U_{0}, & i=3,7,11 \\ U_{0}, & i=4,8,12\end{cases}
$$

and finally we put

$$
\Gamma_{i}= \begin{cases}\frac{1}{C_{w}}, & i=1,2,3,4 \\ 0, & i=5,6, \ldots, 12\end{cases}
$$


At the switching surfaces (the three planes and the cylinder), the vector field is defined through the Filippov convention, cf Filippov [6]. In all simulations the parameter values $R=0.2 \Omega, L=31 \cdot 10^{-6} \mathrm{H}, C=2 \cdot 10^{-6} \mathrm{~F}$, and $C_{w}=$ $0.4 \cdot 10^{-6} \mathrm{~F}$ were used.

If $|z|>U_{0}$, then $\dot{z}=0$, and the system reduces by definition into the two-dimensional system studied in Melin and Hultgren [18]. The application itself makes these cases of limited interest, too. Therefore we assume in the sequel that $|z| \leq U_{0}$. Furthermore, the Filippov convention for defining the vector field at the switching surfaces implies the invariance of this region, ie all trajectories with initial values in $|z| \leq U_{0}$ remain in the region $|z| \leq U_{0}$.

Direct substitution of (4) into (1) yields the following system

$$
\left(\begin{array}{c}
\dot{x} \\
\dot{y} \\
\dot{z}
\end{array}\right)=\left(\begin{array}{c}
y / C \\
-\left(x+R \cdot y+z-u_{i}\right) / L \\
y / C_{w}
\end{array}\right) .
$$

for $|z|<U_{0}$. We assume that all parameters are positive and that the system possesses the resonant property.

$$
\frac{L}{C}+\frac{L}{C_{w}}>\frac{R^{2}}{4} .
$$

Next consider the system outside the switching surfaces. The resonant property implies that the eigenvalues of the system (5) are given by $\lambda_{1}=0$ and

$$
\lambda_{2,3}=-\frac{1}{L}\left(\frac{R}{2} \pm i \sqrt{\frac{L}{C}+\frac{L}{C_{w}}-\frac{R^{2}}{4}}\right)
$$

ie the system possesses a couple of non-real eigenvalues provided the resonant property holds and as far as we remain outside the switching surfaces. It will turn out that, for instance, all fixed points are located at the switching surface $y=0$, so the negative sign of the real part of these eigenvalues does not reveal the stability properties of any fixed points of the system (5).

Remark 1. Using (3) we note that (5) is preserved under the transformation $X=-x, Y=-y, Z=-z$. We have reported corresponding symmetry in the two-dimensional case earlier (Melin and Hultgren [18, 17]). As a consequence, possible limit cycles and possible other types of non-wandering sets of (5) must be preserved or reflected onto other similar co-existing nonwandering sets under the same geometric transformation. 


\section{The trajectories}

It follows from (5) that the fixed points are located at line segments satisfying the relation

$$
(x, y, z)=t \cdot(-1,0,1)+u_{i} \cdot(1,0,0)
$$

where $|t| \leq U_{0}$. Obviously, these line segments can also be expressed through the equation $x+z=u_{i}$ in the $x z$-plane.

We describe the structure of the nonwandering (Guckenheimer and Holmes [7]) sets of (5).

Lemma 1. The nonwandering sets of (5) belong to the following classes:

(A) Fixed points located at (7).

(B) Limit cycles in the planes

$$
z=\frac{C}{C_{w}} \cdot x+D, \text { or }
$$

(C) unions of joined trajectory segments in the pair of planes $|z|=U_{0}$ and the family of planes $z=\frac{C}{C_{w}} \cdot x+D_{j}$, with $j=1,2, \ldots$, where $D_{j}$ are (possibly initial value dependent) constants and $D_{j} \neq 0, j=1,2, \ldots$

Remark 2. Since all fixed points are located in the discontinuity plane $y=0$, their stability are not determined by the sign of the real part of the eigenvalues (6). In fact, we shall prove later that all fixed points in (7) are unstable, so the system can possess attracting sets of type (B) and (C) only.

Remark 3. If $D \neq 0$, and a limit cycle is found, for instance for $D>0$, there must be a corresponding limit cycle also for $D<0$ by symmetri, cf Remark 1.

We shall later elucidate that the structure of the the non-wandering sets of type (C) in more detail. In Section 5 we will elucidate some cases when these non-wandering sets reduce to limit cycles.

Proof. If $|z|<U_{0}$ it follows from (5) that $C \cdot \dot{x}=C_{w} \cdot \dot{z}$, this implies

$$
C \cdot x=C_{w} \cdot z+D,
$$

where $D$ is an initial value dependent constant. Let the initial values be such that $D=D_{1}$. From (1) and (4) we obtain $\dot{z}=0$ for $z=U_{0}$ and $y>0$ 
or $z=-U_{0}$ and $y<0$ implying that $z$ is kept constant at these two halfplanes (note that the Filippov convention was used here). All trajectories that do not remain in any plane of type (9) must enter one of these two half-planes and must leave these half-planes after passing the plane $y=0$ (cf. corresponding analysis of the two-dimensional case, Melin and Hultgren $[17,18])$. After passing the plane $y=0$, the Filippov convention implies that the trajectory must leave the planes $|z|=U_{0}$ and follow a plane of type (9) with a possibly other initial value constant, say $D=D_{2}$. Again, if the trajectory does not remain in the plane, it will approach one of the planes $|z|=U_{0}$ again and leave this plane after approaching the plane $y=0$ again. So in case the trajectory does not remain in any plane on the form (9), we have the case $(\mathrm{C})$. The extended Poincaré-Bendixson theorem [15] prevents the occurrence of more complicated limit sets that are limited to these planes. So, if the trajectory does neither approach a fixed point at (7) nor remain in some plane of form (9), (C) occurs.

\section{Limit cycles inside the cylinder}

First consider trajectories entirely inside the cylinder (Type I). We project the orbits in the planes (8) onto the plane $z=0$ and get

$$
\left(\begin{array}{c}
\dot{x} \\
\dot{y}
\end{array}\right)=\left(\begin{array}{c}
y / C \\
-\left(\left(1+\frac{C}{C_{w}}\right) x+R y-u_{i}+D\right) / L
\end{array}\right) .
$$

A complete analysis of the qualitative behavior of a system quite close to the above one was given by Melin and Hultgren [18]. We shall use existing knowledge concerning the two-dimensional system in order to make some statements regarding the dynamics of the three-dimensional system.

Now we are going to prove existence and uniqueness of limit cycles entirely inside the circle. The switching regions $\Omega_{i}, i=1,2$ are defined by 2 . The fixed points are $\left(\frac{u_{i}-D}{1+\frac{C}{C_{w}}}, 0\right)$ and the eigenvalues $\lambda_{1,2}=-a \pm i \cdot \omega$, where $a=\frac{R}{2 L}$ and $\omega=\sqrt{\frac{1}{L C}+\frac{1}{L C_{w}}-\frac{R^{2}}{4 L^{2}}}$. Consider a trajectory with starting point $P_{0}:\left(r_{0}, 0\right)$, $r_{0}>0$ inside the circle. The system is a forced linear system in each switching region so it can be integrated. The following solution is obtained

$$
\left(\begin{array}{l}
x(t) \\
y(t)
\end{array}\right)=\left(\begin{array}{c}
\frac{u_{i}-D}{1+\frac{C}{C_{w}}}+\left(r_{0}-\frac{u_{i}-D}{1+\frac{C}{C_{w}}}\right) \cdot e^{-a t} \cdot\left(\cos (\omega t)+\frac{a}{\omega} \cdot \sin (\omega t)\right) \\
-\left(r_{0}-\frac{u_{i}-D}{1+\frac{C}{C_{w}}}\right) \cdot \frac{e^{-a t}}{\omega L} \cdot \sin (\omega t)
\end{array}\right)
$$


The trajectory enters $\Omega_{2}$. Using $u_{2}=U_{0}-E$ we obtain the intersection with the negative $x$-axis at $P_{1}:\left(r_{1}, 0\right)$ where

$$
r_{1}=-\frac{E-U_{0}+D}{1+\frac{C}{C_{w}}}-\left(r_{0}+\frac{E-U_{0}+D}{1+\frac{C}{C_{w}}}\right) \cdot e^{-\frac{a \pi}{\omega}}
$$

Let $P_{1}$ be the starting point of the trajectory in $\Omega_{1}$. Using $u_{1}=E-U_{0}$, the trajectory once again intersects the positive $x$-axis. This time at $P_{2}:\left(r_{2}, 0\right)$, where

$$
r_{2}=\frac{E-U_{0}-D}{1+\frac{C}{C_{w}}}-\left(r_{1}-\frac{E-U_{0}-D}{1+\frac{C}{C_{w}}}\right) \cdot e^{-\frac{a \pi}{\omega}}
$$

If we put $r_{1}$ from (11) into (12) we get

$$
r_{2}=\frac{E-U_{0}-D}{1+\frac{C}{C_{w}}}+\frac{2\left(E-U_{0}\right)}{1+\frac{C}{C_{w}}} \cdot e^{-\frac{a \pi}{\omega}}+\left(r_{0}+\frac{E-U_{0}-D}{1+\frac{C}{C_{w}}}\right) \cdot e^{-\frac{2 a \pi}{\omega}}
$$

The trajectory is closed if and only if $r_{2}=r_{0}$, this gives us

$$
r_{0}=\frac{E-U_{0}}{1+\frac{C}{C_{w}}} \cdot \frac{1+e^{-\frac{a \pi}{\omega}}}{1-e^{-\frac{a \pi}{\omega}}}-\frac{D}{1+\frac{C}{C_{w}}}
$$

If we put (13) into (11) we obtain

$$
r_{1}=-\frac{E-U_{0}}{1+\frac{C}{C_{w}}} \cdot \frac{1+e^{-\frac{a \pi}{\omega}}}{1-e^{-\frac{a \pi}{\omega}}}-\frac{D}{1+\frac{C}{C_{w}}}
$$

We summarize our results in a lemma.

Lemma 2. If $\left|E-U_{0}\right|$ is sufficiently small then for every $D$ the system (10) possesses an unique limit cycle entirely inside the circle $x^{2}+y^{2}=i_{r}^{2}$ passing through the points $P_{0}$ and $P_{1}$ where $r_{0}$ and $r_{1}$ are defined by (13) respectively (14).

Now let us return to the three-dimensional system (5) and consider first the plane $(8)$ through origin $(D=0)$. In this plane we have an unique limit cycle through the points

$$
\left(\frac{E-U_{0}}{1+\frac{C}{C_{w}}} \cdot \frac{1+e^{-\frac{a \pi}{\omega}}}{1-e^{-\frac{a \pi}{\omega}}}, \quad 0, \quad \frac{C}{C_{w}} \cdot \frac{E-U_{0}}{1+\frac{C}{C_{w}}} \cdot \frac{1+e^{-\frac{a \pi}{\omega}}}{1-e^{-\frac{a \pi}{\omega}}}\right)
$$

and

$$
\left(-\frac{E-U_{0}}{1+\frac{C}{C_{w}}} \cdot \frac{1+e^{-\frac{a \pi}{\omega}}}{1-e^{-\frac{a \pi}{\omega}}}, \quad 0, \quad-\frac{C}{C_{w}} \cdot \frac{E-U_{0}}{1+\frac{C}{C_{w}}} \cdot \frac{1+e^{-\frac{a \pi}{\omega}}}{1-e^{-\frac{a \pi}{\omega}}}\right)
$$


See Figures 5-6 $(*$-marks represent the fixed points). Note that arrows in some of the figures represent the directions of the trajectories.

If the plane (8) moves to the right, then $D$ decreases. For every $D$ there exists an unique limit cycle, in (8), through the points $\left(r_{0}, 0, \frac{C}{C_{w}} \cdot r_{0}+D\right)$ and $\left(r_{1}, 0, \frac{C}{C_{w}} \cdot r_{1}+D\right)$ where $r_{0}$ and $r_{1}$ are defined by (13) respectively (14). This holds until the limit cycle intersects the plane $z=-U_{0}$. In that case we have $-U_{0}=\frac{C}{C_{w}} \cdot r_{1}+D$. If we put (14) into this formula we obtain

$$
D=D_{0}=\frac{C}{C_{w}} \cdot\left(E-U_{0}\right) \cdot \frac{1+e^{-\frac{a \pi}{\omega}}}{1-e^{-\frac{a \pi}{\omega}}}-\left(1+\frac{C}{C_{w}}\right) \cdot U_{0}
$$

For every plane (8) there is a corresponding symmetric plane

$$
z=\frac{C}{C_{w}} \cdot x-D
$$

with corresponding limit cycles passing through the points $\left(-r_{0}, 0,-\frac{C}{C_{w}} \cdot r_{0}-D\right)$ and $\left(-r_{1}, 0,-\frac{C}{C_{w}} \cdot r_{1}-D\right)$ where $r_{0}$ and $r_{1}$ are defined by (13) respectively (14). The symmetry of the limit cycles are satisfied according to Remark 1 see Figures 3-4. If the plane (8) moves further to the right $\left(D<D_{0}\right)$, a possible limit cycle must pass a point $\left(r_{0}, 0, U_{0}\right)$. This implies $U_{0}=\frac{C}{C_{w}} \cdot r_{0}+D$ replace $r_{0}$ by (13), we obtain $D=-D_{0}$ which is a contradiction. Consequently there are no limit cycles in this region. In fact every trajectory that starts in this region moves towards the plane $z=-U_{0}$ and follows this plane until it intersects the plane (8) where $D=D_{0}$. The trajectory then spirals into an unique limit cycle through the points

$$
\left(\left(E-U_{0}\right) \cdot \frac{1-\frac{C}{C_{w}}}{1+\frac{C}{C_{w}}} \cdot \frac{1+e^{-\frac{a \pi}{\omega}}}{1-e^{-\frac{a \pi}{\omega}}}+U_{0}, \quad 0, \frac{2 \cdot \frac{C}{C_{w}}}{1+\frac{C}{C_{w}}} \cdot\left(E-U_{0}\right) \cdot \frac{1+e^{-\frac{a \pi}{\omega}}}{1-e^{-\frac{a \pi}{\omega}}}-U_{0}\right)
$$

and

$$
\left(-\left(E-U_{0}\right) \cdot \frac{1+e^{-\frac{a \pi}{\omega}}}{1-e^{-\frac{a \pi}{\omega}}}, \quad 0, \quad-U_{0}\right)
$$

Corresponding phenomena occurs to the left of the plane $z=\frac{C}{C_{w}} \cdot x-D_{0}$. We have proved the following theorem, concerning limit cycles entirely inside the cylinder.

Theorem 1. If $\left|E-U_{0}\right|$ is sufficiently small, then the following holds i) If $D=0$ then at the plane through origin there is an unique limit cycle through the points (15) and (16). 
ii) If $0<|D| \leq\left|D_{0}\right|$ then for every $D$ there is an unique limit cycle at the plane (8) and a corresponding symmetric limit cycle at the plane (18).

iii) If $|D|>\left|D_{0}\right|$ then there are no limit cycles, but every trajectory which starts in this region converges to an unique limit cycle in one of the planes $z=\frac{C}{C_{w}} \cdot x \pm D_{0}$, where $D_{0}$ is defined by $(17)$.

\section{Limit cycles partly outside the cylinder}

Let us consider trajectories which are located partly outside the cylinder. Such a trajectory must at some point intersect a point $\left(r_{0}, 0, U_{0}\right)$, where $r_{0}>i_{r}$. The trajectory follows some plane (8) until it intersects $z=-U_{0}$ at $\left(x_{0}, y_{0},-U_{0}\right)$. From (8) we obtain

$$
r_{0}-x_{0}=2 \cdot \frac{C_{w}}{C} \cdot U_{0}
$$

where $x_{0}>i_{r}$ and $y_{0}<0$. The trajectory stays in the plane $z=-U_{0}$ and moves to the left until it intersects the plane $y=0$. Then it is located in some plane (8) until it intersects the plane $z=U_{0}$ and moves to the right until it once again intersects $y=0$, see Figure 7 .

A projection of such a trajectory in this three-dimensional case onto the $x y$-plane is unique, and a trajectory in the $x y$-plane partly located outside the circle has an unique corresponding trajectory in the three-dimensional case. We summarize this in a Lemma.

Lemma 3. Consider a trajectory of type (C) in Lemma 1. If its corresponding planes (8) are located entirely outside the cylinder, then there is an one-to-one correspondence between this trajectory and its projection at the $x y$-plane.

Now we are going to prove i) existence and ii) uniqueness of limit cycles by first considering the planar case. Let us consider the projection.

i) The intersection point $\left(x_{0}, y_{0}\right)$ between the trajectory and the plane $z=$ $-U_{0}$ is located in the region $\Omega_{4}$. Divide this region into two sub regions $\Omega_{4}^{\prime}$ and $\Omega_{4}^{\prime \prime}$, where $x>x_{0}$ in $\Omega_{4}^{\prime}$ and $x<x_{0}$ in $\Omega_{4}^{\prime \prime}$. We obtain the following system in $\Omega_{4}^{\prime}$ :

$$
\left(\begin{array}{l}
\dot{x} \\
\dot{y}
\end{array}\right)=\left(\begin{array}{c}
y / C \\
-\frac{\left(1+\frac{C}{C_{w}}\right) \cdot x+R \cdot y-\frac{C}{C_{w}} \cdot r_{0}}{L}
\end{array}\right)
$$


Choose a starting point $\left(r_{0}, 0\right)$, where $r_{0}>x_{0}$. Using (19) we obtain the following solution:

$$
\left(\begin{array}{c}
x(t) \\
y(t)
\end{array}\right)=\left(\begin{array}{c}
\frac{r_{0}}{1+\frac{C}{C_{w}}} \cdot\left(\frac{C}{C_{w}}+e^{-a \cdot t}\left(\cos (\omega t)+\frac{a}{\omega} \cdot \sin (\omega \cdot t)\right)\right) \\
-\frac{r_{0}}{\omega \cdot L} \cdot e^{-a \cdot t} \cdot \sin (\omega \cdot t)
\end{array}\right)
$$

Let $t_{0}$ denote the time of intersection, that is $x\left(t_{0}\right)=x_{0}$, together with (19) we get:

$$
r_{0}=\frac{2\left(1+\frac{C_{w}}{C}\right) \cdot U_{0}}{1-e^{-a \cdot t_{0}}\left(\cos \left(\omega \cdot t_{0}\right)+\frac{a}{\omega} \cdot \sin \left(\omega \cdot t_{0}\right)\right)}
$$

The time of intersection $t_{0}$ is very small. In our simulations we can estimate $t_{0}=1.6 \cdot 10^{-6}$. According to this fact we obtain:

$$
r_{0}=\frac{4 \cdot L \cdot C_{w} \cdot U_{0}}{t_{0}^{2}} \cdot\left(1+o\left(t_{0}\right)\right)
$$

Using $(21)$ in $y\left(t_{0}\right)=y_{0}$ we get:

$$
y_{0}=-\frac{4 \cdot C_{w} \cdot U_{0}}{t_{0}}\left(1+o\left(t_{0}\right)\right)
$$

In $\Omega_{4}^{\prime \prime}$ we have the system:

$$
\left(\begin{array}{c}
\dot{x} \\
\dot{y}
\end{array}\right)=\left(\begin{array}{c}
y / C \\
-\frac{x+R \cdot y-2 \cdot U_{0}}{L}
\end{array}\right)
$$

Choose a virtual starting point $\left(r_{0}^{\prime}, 0\right)$, so that the solution of $(24)$ intersects $\left(x_{0}, y_{0}\right)$. The solution then becomes:

$$
\left(\begin{array}{l}
x(t) \\
y(t)
\end{array}\right)=\left(\begin{array}{c}
2 \cdot U_{0}+\left(r_{0}^{\prime}-2 \cdot U_{0}\right) \cdot e^{-a \cdot t} \cdot\left(\cos (b \cdot t)+\frac{a}{b} \cdot \sin (b \cdot t)\right) \\
-\frac{r_{0}^{\prime}-2 \cdot U_{0}}{b \cdot L} \cdot e^{-a \cdot t} \cdot \sin (b \cdot t)
\end{array}\right)
$$

The righthand side of (20) and (24) coinsides at $\left(x_{0}, y_{0}\right)$ so the projection of the trajectory is $C^{1}$ at this point. Consider a tangent line of this projection at $\left(x_{0}, y_{0}\right)$ intersecting the $x$-axis at $\left(x_{01}, 0\right)$, where $x_{0}<r_{0}<x_{01}$ and $x_{0}<$ $r_{0}^{\prime}<x_{01}$. Using this in (24) we obtain:

$$
x_{01}-x_{0}=\frac{\frac{L}{C} \cdot y_{0}^{2}}{r_{0}+R \cdot y_{0}-2 \cdot\left(1+\frac{C_{w}}{C}\right) \cdot U_{0}}
$$


Using (22) and (23) in (26) we obtain:

$$
x_{01}-x_{0}=4 \cdot \frac{C_{w}}{C} \cdot U_{0} \cdot\left(1+o\left(t_{0}\right)\right)
$$

then

$$
r_{0}^{\prime}-r_{0}<x_{01}-x_{0}<4 \cdot \frac{C_{w}}{C} \cdot U_{0}
$$

when $r_{0}$ is large enough. The capacitor $C_{w}$ is a parasite capacitor and we wish it to vanish, but this is not possible according to physics. In our model however we choose it as small as possible. In our simulations we have $C_{w} / C=0.2$. From (27) we obtain $r_{0}^{\prime}-r_{0}<2 \cdot U_{0}$ if $C_{w} / C<0.5$, and as a consequence $r_{0}^{\prime}<r_{0}+2 \cdot U_{o}$. The solution (25) intersects the negative $x$-axis at $r_{1}=2 \cdot U_{0}-\left(r_{0}^{\prime}-2 \cdot U_{0}\right) \cdot e^{-\frac{a \cdot \pi}{b}}=2 \cdot U_{0} \cdot\left(1+e^{-\frac{a \cdot \pi}{b}}\right)-r_{0}^{\prime} \cdot e^{-\frac{a \cdot \pi}{b}}>$ $2 \cdot U_{0} \cdot\left(1+e^{-\frac{a \cdot \pi}{b}}\right)-\left(r_{0}+2 \cdot U_{0}\right) \cdot e^{-\frac{a \cdot \pi}{b}}=2 \cdot U_{0}-r_{0} \cdot e^{-\frac{a \cdot \pi}{b}}>-r_{0}$.

This means that this planar system is dissipative, according to the symmetry and the fact that $\left(r_{0}, 0\right)$ is the real starting point of this system. Let $r_{0}$ decrease until the trajectory $\gamma$ tangents the circle, somewhere in the upper half plane. Let $\left(r_{2}, 0\right)$ be the returning point at the $x$-axis of $\gamma$, then $r_{2}<r_{0}$. Construct a transversal from $\left(r_{2}, 0\right)$ along the $x$-axis to $\left(r_{0}, 0\right)$. This transversal together with the trajectory $\gamma$ encloses a compact region $K$. According to the extended Poincaré-Bendixson theorem [15], there exists a closed trajectory in $K$ and this trajectory intersects the circle. The dissipativity implies that there is no closed orbit entirely outside the circle. Such a region $K$ is always possible to construct if there are no fixed points outside the circle, but it could be possible anyway. We will give some more comments on this at the end of this section.

ii) Let us consider the uniqueness of such a limit cycle. The characteristic functions $\chi_{\Omega}$ of the regions $\Omega$, can be expressed in terms of Heavyside functions:

$$
\begin{aligned}
& \chi_{\Omega_{1}}=\left(1-H\left(x^{2}+y^{2}-i_{r}^{2}\right)\right) \cdot H(y) \\
& \chi_{\Omega_{2}}=\left(1-H\left(x^{2}+y^{2}-i_{r}^{2}\right)\right) \cdot(1-H(y)) \\
& \chi_{\Omega_{3}^{\prime}}=H\left(x^{2}+y^{2}-i_{r}^{2}\right) \cdot\left(1-H\left(x+x_{0}\right)\right) \cdot H(y) \\
& \chi_{\Omega_{3}^{\prime \prime}}=H\left(x^{2}+y^{2}-i_{r}^{2}\right) \cdot H\left(x+x_{0}\right) \cdot H(y) \\
& \chi_{\Omega_{4}^{\prime}}=H\left(x^{2}+y^{2}-i_{r}^{2}\right) \cdot H\left(x-x_{0}\right) \cdot(1-H(y)) \\
& \chi_{\Omega_{4}^{\prime \prime}}=H\left(x^{2}+y^{2}-i_{r}^{2}\right) \cdot\left(1-H\left(x-x_{0}\right)\right) \cdot(1-H(y))
\end{aligned}
$$


The corresponding righthand sides $f_{i}$ of the system (10) are:

$$
\begin{aligned}
& f_{1}=\left(\begin{array}{c}
y / C \\
-\frac{x+R \cdot y-E+2 \cdot U_{0}}{L}
\end{array}\right),(x, y) \in \Omega_{1} \\
& f_{2}=\left(\begin{array}{c}
y / C \\
-\frac{x+R \cdot y+E-2 \cdot U_{0}}{L}
\end{array}\right),(x, y) \in \Omega_{2} \\
& f_{3}^{\prime}=\left(\begin{array}{c}
y / C \\
-\frac{\left(1+\frac{c}{C_{w}}\right) \cdot x+R \cdot y+\frac{C}{C_{w}} \cdot r_{0}}{L}
\end{array}\right),(x, y) \in \Omega_{3}^{\prime} \\
& f_{3}^{\prime \prime}=\left(\begin{array}{c}
y / C \\
-\frac{x+R \cdot y+2 \cdot U_{0}}{L}
\end{array}\right),(x, y) \in \Omega_{3}^{\prime \prime} \\
& f_{4}^{\prime}=\left(\begin{array}{c}
y / C \\
-\frac{\left(1+\frac{C}{C_{w}}\right) \cdot x+R \cdot y-\frac{C}{C_{w}}}{L} \cdot r_{0}
\end{array}\right),(x, y) \in \Omega_{4}^{\prime} \\
& f_{4}^{\prime \prime}=\left(\begin{array}{c}
y / C \\
-\frac{x+R \cdot y-2 \cdot U_{0}}{L}
\end{array}\right),(x, y) \in \Omega_{4}^{\prime \prime}
\end{aligned}
$$

The righthand side $f$ of (10) can be written as:

$$
f=f_{1} \cdot \chi_{\Omega_{1}}+f_{2} \cdot \chi_{\Omega_{2}}+f_{3}^{\prime} \cdot \chi_{\Omega_{3}^{\prime}}+f_{3}^{\prime \prime} \cdot \chi_{\Omega_{3}^{\prime \prime}}+f_{4}^{\prime} \cdot \chi_{\Omega_{4}^{\prime}}+f_{4}^{\prime \prime} \cdot \chi_{\Omega_{4}^{\prime \prime}}
$$

We can rewrite this as following:

$f=f_{2}+\left(f_{1}-f_{2}+\left(f_{3}^{\prime \prime}-f_{3}^{\prime}\right) \cdot H\left(x+x_{0}\right)\right) \cdot H(y)+$ $\left(f_{4}^{\prime \prime}-f_{2}+\left(f_{4}^{\prime}-f_{4}^{\prime \prime}\right) \cdot H\left(x-x_{0}\right)\right) \cdot H\left(x^{2}+y^{2}-i_{r}^{2}\right)+$ $\left(f_{2}-f_{1}+f_{3}^{\prime}-f_{4}^{\prime \prime}+\left(f_{4}^{\prime \prime}-f_{4}^{\prime}\right) \cdot H\left(x-x_{0}\right)\right) \cdot H(y) \cdot H\left(x^{2}+y^{2}-i_{r}^{2}\right)$

When we calculate the divergence we obtain the following formula:

$$
\operatorname{div} f(x, y)=\operatorname{div} f_{2}(x, y)+c_{1}(x, y) \cdot \delta(y)+c_{2}(x, y) \cdot \delta\left(x^{2}+y^{2}-i_{r}^{2}\right)
$$

After some calculations we obtain the following:

1 ) the divergence of the continuous part is $\operatorname{div} f_{2}(x, y)=-R / L<0$.

$2)$ if $(\bar{x}, \bar{y})$ is a discontinuity point at the $x$-axis then $c_{1}(\bar{x}, \bar{y})=-\frac{2 \cdot U_{0}}{L}$.

$3)$ if $(\bar{x}, \bar{y})$ is a discontinuity point at the circle then $c_{2}(\bar{x}, \bar{y})=-\frac{2 \cdot E \cdot|y|}{L}$.

Note that the intersection points $\left(x_{0}, y_{0}\right)$ and $\left(-x_{0},-y_{0}\right)$ cause no Dirac impulses. The coefficients $c_{1}$ and $c_{2}$ are negative at its respectively discontinuity points. This implies that the Floquet exponent $\mu$ of this limit cycle is negative. By the extended theorem concerning Floquet exponents in [14] we 
state that every limit cycle of this type is asymptotically stable and unique. Similar calculations are considered in [14] and [18].

This concludes the planar case. Let us return to the three-dimensional case. According to Lemma 3, there exists a corresponding three-dimensional limit cycle which is asymptotically stable and unique. We have proved the following theorem concerning limit cycles intersecting the cylinder.

Theorem 2. If $\left|U_{0}\right|$ is sufficiently small, then there exists an unique limit cycle of (5) intersecting the cylinder.

Note that $2 \cdot\left|U_{0}\right|<i_{r}$ means that the system (5) has no fixed points outside the cylinder, so in that case the theorem is applicable. Theorem 2 is applicable even if $2 \cdot\left|U_{0}\right| \geq i_{r}$ if $\left|U_{0}\right|$ is small enough. Such cases are considered in [18].

In this paper we refer to two types of limit cycles, where existence and uniqueness are proved in a quite different way.

Type I: entirely inside the cylinder and Type II: intersecting the cylinder.

A limit cycle of Type II is shown in Figures 8-9.

\section{Summary}

In this paper the existence and uniqueness of a limit cycle in a three-dimensional approximation of a resonant converter was proved for a number of important special cases. Our idea was to project certain trajectories of the system onto a plane and use results proved earlier by Melin and Hultgren [18] for a twodimensional approximation of the resonant converter modelled here. This study has been focused at analysis of possible limit cycles of resonant converter system and our study does not exclude more complicated dynamical behavior. As we simulated the system in order to illustrate the limit cycles we encountered a number of numerical problems. In the two-dimensional case numerical problem appeared when the fixed points were located outside the circle and an interval of fixed points at the $x$-axis caused so called chattering. This phenomenon gave rise to limit cycles that exist in the numerical study only, whereas our analysis indicated that these trajectories were absorbed by attracting sliding modes in the switching regions. This justifies careful analytical studies of dynamical behavior of the resonant converter, since these numerical problems grow essentially in higher dimensional cases because the 
set of fixed points increases. We avoided these problems by carefully preparing our numerical experiments with respect to the bifurcation parameters. Also higher dimensional approximations of the resonant converter has been suggested, see Hultgren and Lenells [11]. Already completing the analysis of the three-dimensional system to include cases left open here may turn out to be very difficult. Analysis of the special case considered here was possible, because of the symmetry and the fact that the trajectories between the "top" and the "bottom" are located in certain planes. The time the trajectories spend at these planes is very short compared to the time spend at the "top" and the "bottom". Thus, we could name the cases considered here "almost planar cases".

It is possible that analyzing existence and uniqueness of limit cycles are impossible without the symmetry properties, in any case the calculations will be more extensive depending on which nonsymmetric system we are supposed to deal with.

From application point of view, one aim with the presented study is to reveal potential problems in the suggested control strategy. Theorem 1 states that for certain parameter values there exists non unique limit cycles, implying that special care must be taken in the development of control laws for the resonant converter. The effects of reachable non unique limit cycles must be studied and probably measures must be taken to avoid them.

When implementing the controller normally a computer is used, giving a time discrete system. The switching curves are preferably given in tables. The sampling interval must be short enough and the table quantization must be small enough. The sampling can give anomaly effects especially when the system trajectory passes the switching curves. For discussions of implementation of a switched observer and a switched controller see Hultgren et al (2000) [2] and Hultgren et al (2002) [1].

\section{References}

[1] M. L. P. I. A. Hultgren, P. Ranstad and M. Nilsson. Switched controllers applied to a lcc-converter. In Proceedings of NOR/pie, Stockholm, Sweden, August 12-14. IEEE and PELS, 2002.

[2] W. K. A. Hultgren and M. Lenells. Switched kalman filter in a high frequency series loaded resonant converter. In Proceedings of the IEEE 
Conference on Control Applications, pages 507-512. IEEE, 2000.

[3] P. R. D. Flieller and J. Louis. Computation and stability of limit cycles in hybrid systems. Nonlinear Analysis, 64:352-367, 2006.

[4] M. diBernardo and K. Camlibel. Structural stability of boundary equilibria in a class of hybrid systems: Analysis and use for control system design. In Proceedings of the 44th IEEE Conference on Decision and Control, and the European Control Conference, Seville, Spain, December 12-15. IEEE, 2005.

[5] E. F. F. Angulo, M. diBernardo and G. Olivar. Feedback control of limit cycles: A switching control strategy based on nonsmooth bifurcation theory. IEEE Transactions on Circuits and Systems, 52(2):366-378, 2005 .

[6] A. Filippov. Differential Equations with Discontinuous Righthand Sides. Kluwer Academic Publisher, 1988.

[7] J. Guckenheimer and P. Holmes. Nonlinear Oscillations, Dynamical Systems, and Bifurcations of Vector Fields. Springer-Verlag, 1983.

[8] M. W. Hirsch and S. Smale. Differential Equations, Dynamical Systems, and Linear Algebra. Academic Press, New York, 1974.

[9] L. Hörmander. The Analysis of Linear Partial Differential Operators I. Springer, 1990.

[10] Z. Hou. Bendixsonś criterion for systems with non-smooth vector fields. In Proceedings of the Royal Society of Edinburgh,133A, pages 393-407, 2003.

[11] A. Hultgren and M. Lenells. Stability of a switched hamiltonian observer applied to a resonant converter. In Proceedings of the IEEE PESC 04, Aachen, Germany, 20-25 June. IEEE, 2004.

[12] M. Y. Li and J. S. Muldowney. A geometric approach to global-stability problems. SIAM Journal of Mathematical Analysis, 27(4):1070-1083, 1996.

[13] Y. Li and J. S. Muldowney. On Bendixson's Criterion. Journal of Differential Equations, 106:27-39, 1993. 
[14] J. Melin. Upper bounds for the number of limit cycles of switched systems through distribution theory. International Journal of Hybrid Systems, 3(4):331-340, 2003.

[15] J. Melin. Does distribution theory contain means for extending PoincaréBendixson theory? Journal of Mathematical Analysis and Applications, 303:81-89, 2004.

[16] J. Melin. Some examples describing the need of modifying the Bendixson criterion for piecewise $C^{1}$ - system. Nonlinear Analysis: Theory, Methods and Applications, 62(8):1516-1526, 2005.

[17] J. Melin and A. Hultgren. A limit cycle of a resonant converter. In H. G. S. Engell and J. Zaytoon, editors, Analysis and design of hybrid systems 2003, pages 169-174. IFAC, Elsevier, 2003.

[18] J. Melin and A. Hultgren. On conditions for regularity of solutions for a piecewise linear system. Journal of Nonlinear Analysis: Hybrid Systems and Applications, 65(12):2277-2301, 2006.

[19] H. L. Smith. Monotone dynamical systems : an introduction to the theory of competitive and cooperative systems. Providence, R.I. : American Mathematical Society, 1995.

[20] X.-S. Yang and G. Chen. Limit cycles and chaotic invariant sets in autonomous hybrid planar systems. Nonlinear Analysis: Hybrid Systems, 2:952-957, 2008.

[21] Ye Y.-Q. et al. Theory of Limit Cycles. American Mathematical Society, second edition, 1986.

[22] Zhang Z.-F., Ding T.-R., Huang W.-Z, and Dong Z.-X. Qualitative theory of differential equations. American Mathematical Society, 1992. 


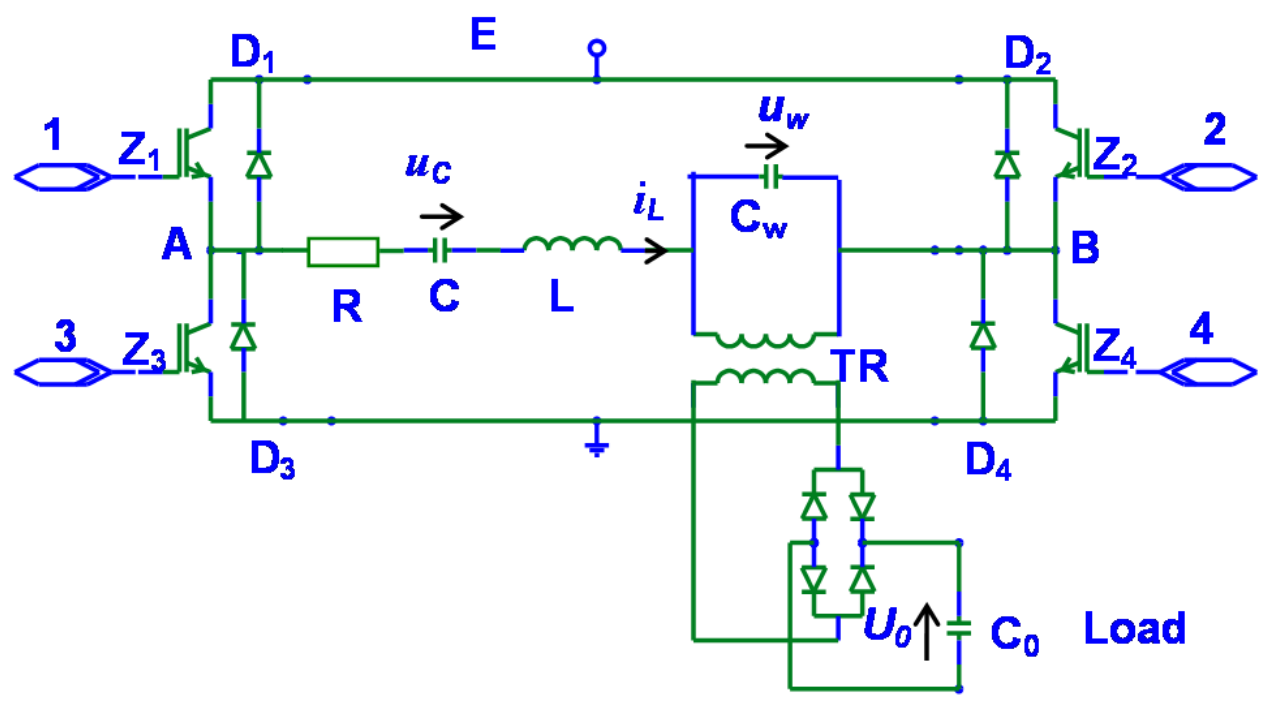

Figure 1: Electrical circuit

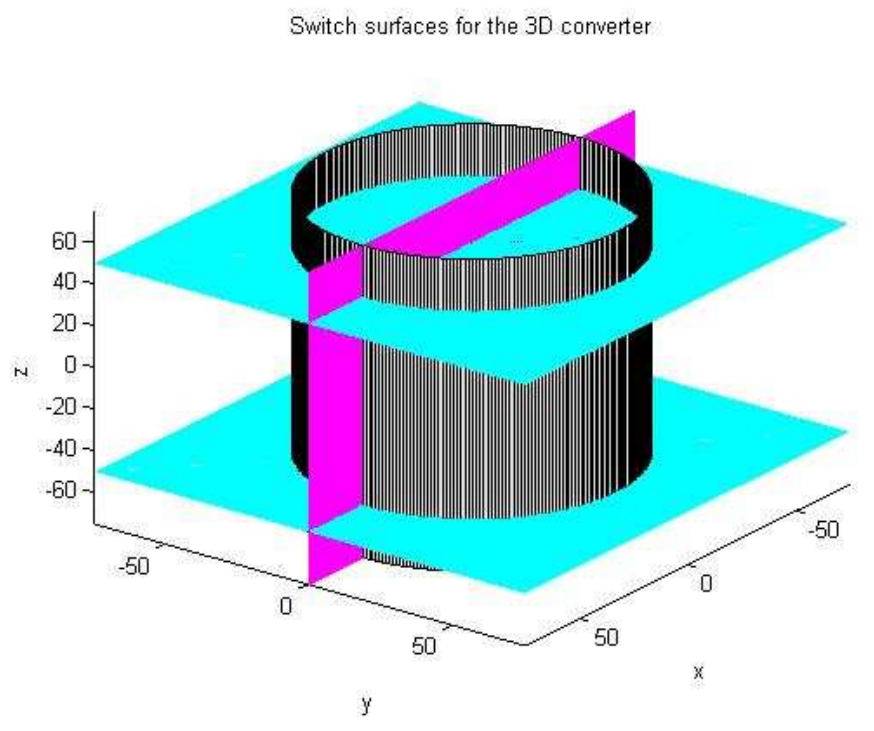

Figure 2: Switching surfaces 


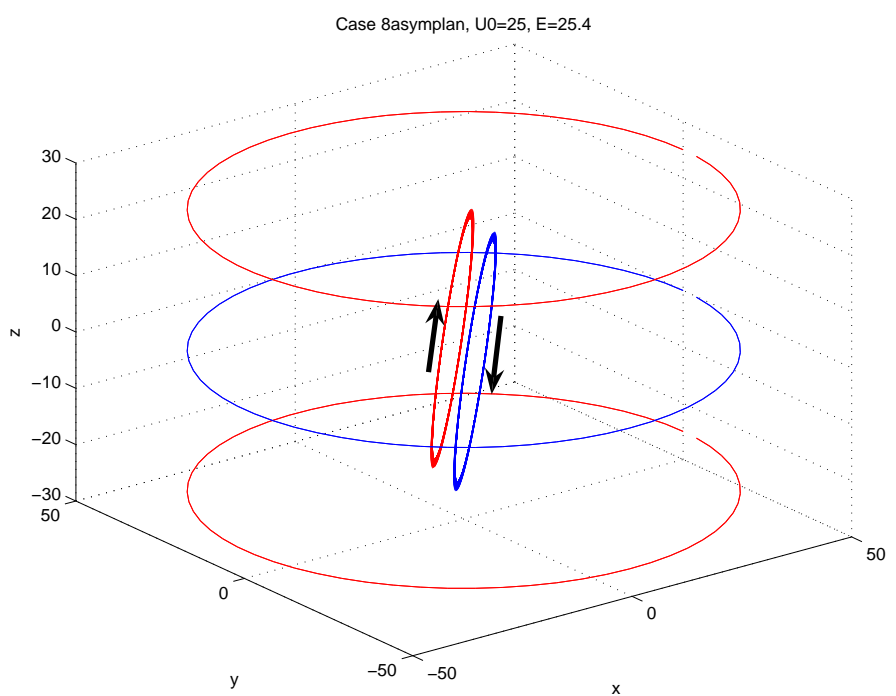

Figure 3: Symmetric limit cycles type I

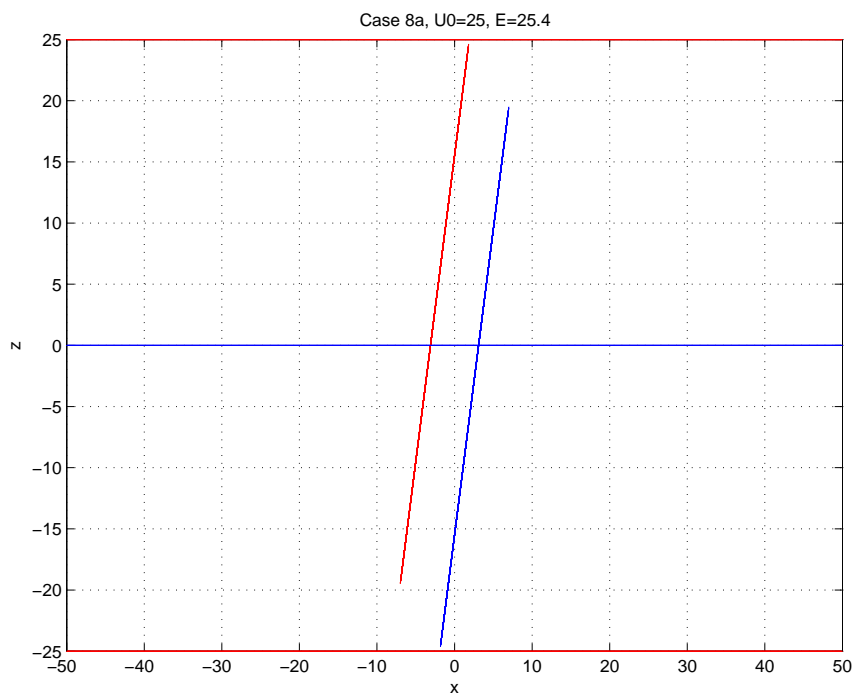

Figure 4: Symmetric limit cycle type I, xz-projection 


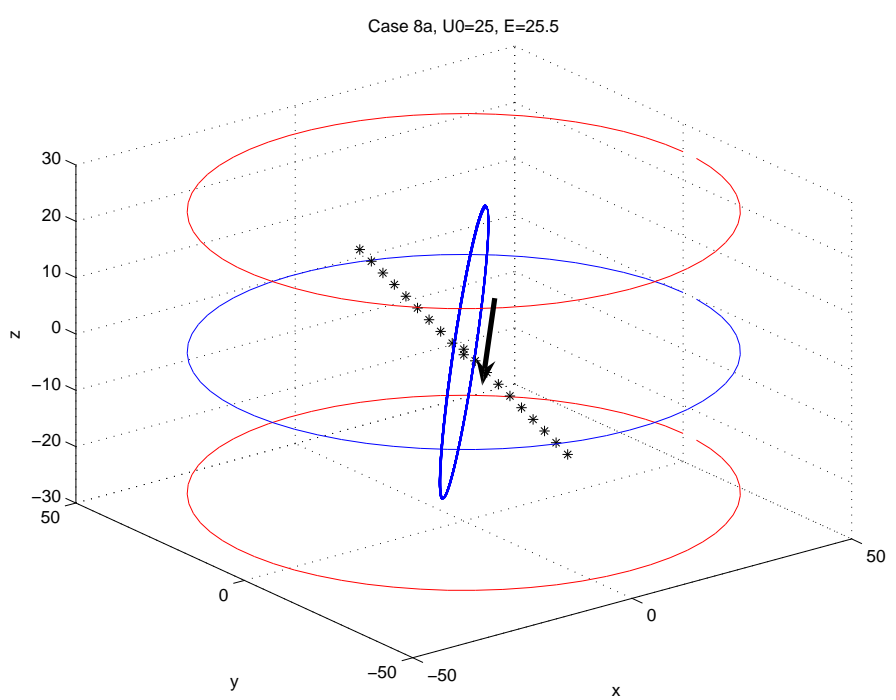

Figure 5: Limit Cycle Type I

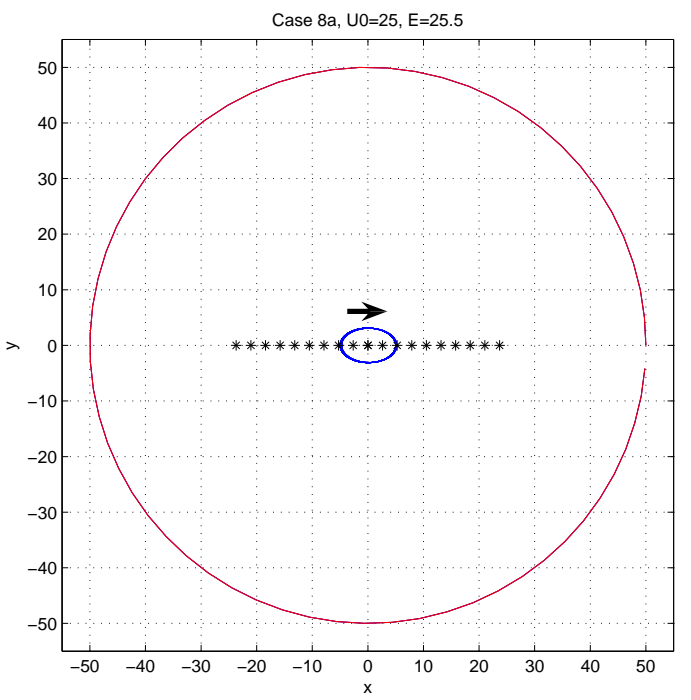

Figure 6: Limit Cycle Type I, xy-projection 


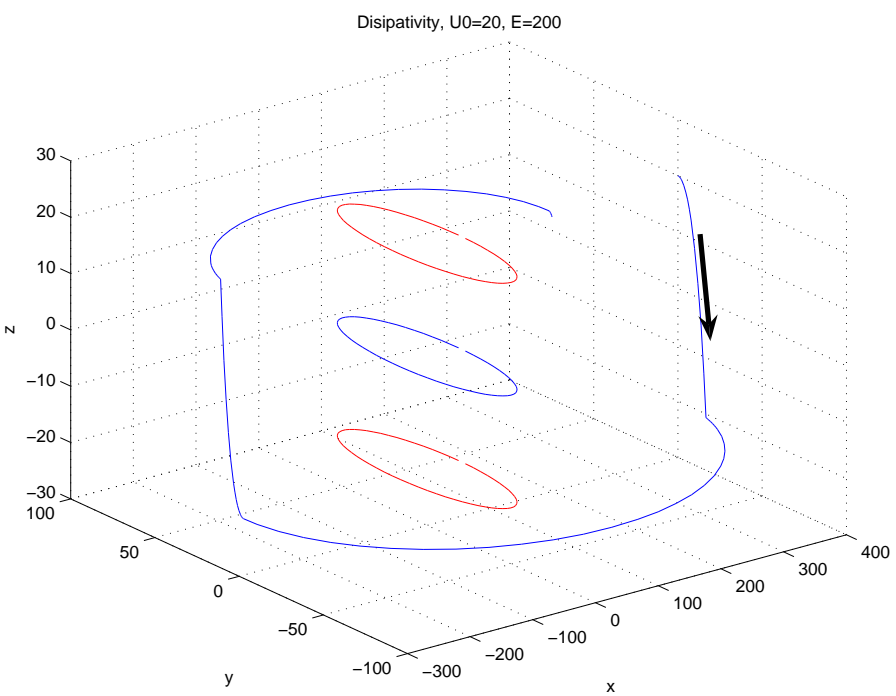

Figure 7: Trajectory outside the cylinder

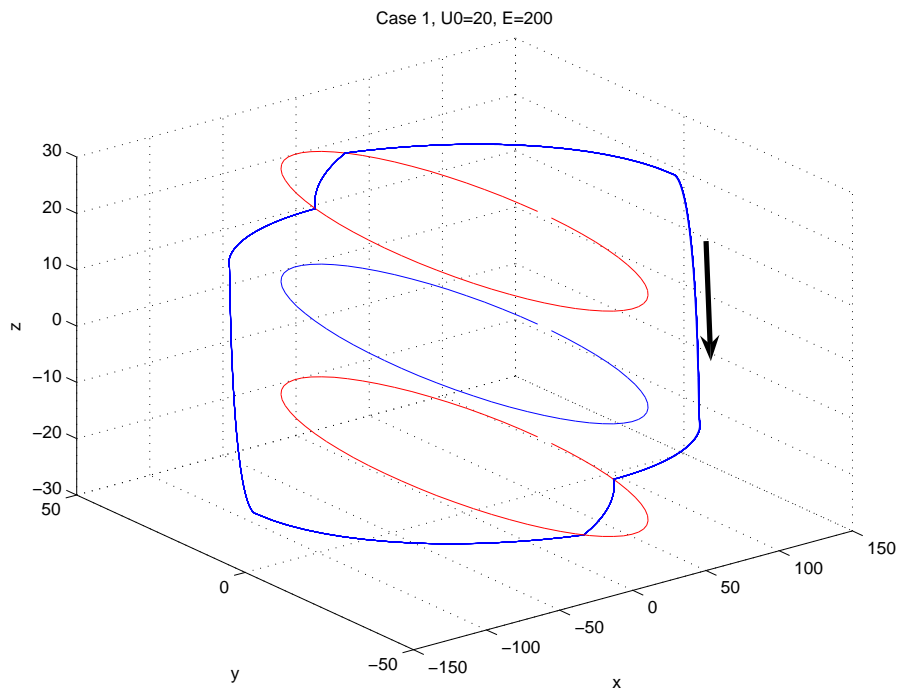

Figure 8: Limit Cycle Type II 


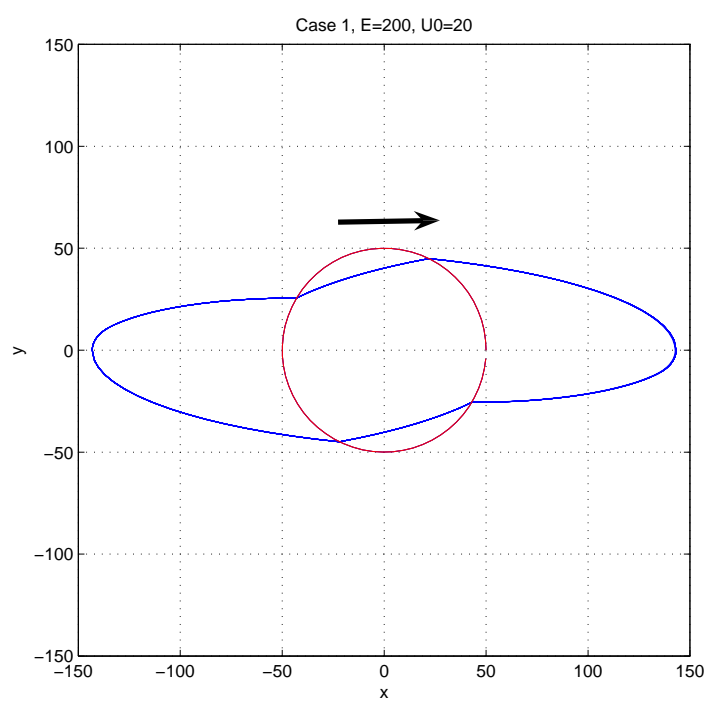

Figure 9: Limit Cycle Type II, xy-projection 